\title{
Dendritic cell populations in patients with self-reported food hypersensitivity
}

This article was published in the following Dove Press journal:

International Journal of General Medicine

13 May 201 I

Number of times this article has been viewed

\author{
Gülen A Lied 1,3,4,* \\ Petra Vogelsang 2 ,* \\ Arnold Berstad ${ }^{1,4}$ \\ Silke Appel ${ }^{2}$ \\ 'Institute of Medicine, ${ }^{2}$ Broegelmann \\ Research Laboratory, The \\ Gade Institute, University of \\ Bergen, Norway; ${ }^{3}$ Division of \\ Gastroenterology, Department \\ of Medicine; ${ }^{4}$ Section of Clinical \\ Allergology, Department of \\ Occupational Medicine, Haukeland \\ University Hospital, Bergen, Norway \\ *These authors contributed equally \\ to this work
}

\begin{abstract}
Self-reported hypersensitivity to food is a common condition and many of these patients have indications of intestinal immune activation. Dendritic cells (DCs) are recognized as the most potent antigen-presenting cells involved in both initiating immune responses and maintaining tolerance. The aims of this study were to evaluate the DC populations with their phenotype and $\mathrm{T}$ cell stimulatory capacity in patients with food hypersensitivity and to study its relationship with atopic disease. Blood samples from 10 patients with self-reported food hypersensitivity, divided into atopic and nonatopic subgroups, and 10 gender- and age-matched healthy controls were analyzed by flow cytometry using the Miltenyi Blood Dendritic cells kit. Monocyte-derived DCs (moDCs) were evaluated concerning their phenotype and T cell stimulatory capacity. DC populations and cell surface markers were not significantly different between patients and healthy controls, but moDCs from atopic patients expressed significantly more CD38 compared to moDCs from nonatopic patients. Moreover, lipopolysaccharide stimulated moDCs from atopic patients produced significantly more interleukin-10 compared to nonatopic patients. CD38 expression was correlated to total serum immunoglobulin E levels. These findings support the notion of immune activation in some patients with self-reported food hypersensitivity. They need to be confirmed in a larger cohort.
\end{abstract}

Keywords: food hypersensitivity, atopy, dendritic cells, CD38

\section{Introduction}

Self-reported hypersensitivity to food is common in the general population, but there is a big discrepancy between perceived food hypersensitivity (20\%) and medically confirmed food allergy $(3 \%-5 \%) .{ }^{1}$ Food allergy can be immunoglobulin E (IgE)-mediated (immediate, type I reactions) or non-IgE-mediated (delayed-type hypersensitivity, type III or IV). ${ }^{2}$ Medically confirmed allergic reactions are usually IgE-mediated, but non-IgE-mediated (cell-mediated) reactions also exist and are more difficult to diagnose. Gastrointestinal symptoms, especially diarrhea, abdominal pain, and bloating, are among the most common complaints attributed to food hypersensitivity. In our previous studies, the majority of patients with self-reported food hypersensitivity had irritable bowel syndrome (IBS). ${ }^{3}$ And as in patients with IBS, administration of low-digestible carbohydrates often replicated their gastrointestinal symptoms. ${ }^{4}$ However, the underlying pathophysiological mechanisms of the IBS-like complaints in patients with food hypersensitivity are unknown so far, and there are no current standard diagnostic methods available for diagnosing non-IgE-mediated delayed-type hypersensitivity reactions, which remain as a possible cause in many of the patients. ${ }^{5}$
Correspondence: Gülen Arslan Lied Section of Gastroenterology, Department of Medicine, Haukeland University Hospital, N-502I, Bergen, Norway

Tel +4755972130

Fax +4755974973

Email gulen.arslan@med.uib.no 
The mucosa of the gastrointestinal tract is constantly in contact with food proteins and bacteria. Dietary protein antigens interact with specific antigen-presenting cells (APCs), which help to activate regulatory $\mathrm{T}$ cells, usually resulting in a suppression of immune responses. ${ }^{6}$ Dendritic cells (DCs) are recognized as the most potent APCs with the unique ability to initiate and maintain primary immune responses. ${ }^{7}$ DCs acquire antigens in the periphery and migrate to the lymph nodes where antigen-specific $\mathrm{T}$ lymphocytes recognize the presented peptide antigens and mount an immune response. ${ }^{78}$ Moreover, DCs are pivotal in inducing tolerance. Initially it was thought that this was restricted to immature DC that present self-antigens in the absence of an additional stimulus to circulating naïve $T$ cells in peripheral lymphoid organs. In the case of autoreactivity, this will normally lead to anergy or deletion of auto-reactive T cells, thereby inducing tolerance. ${ }^{9,10}$ However, this view was challenged when it was shown that also DCs with a mature phenotype are able to expand regulatory T cells (Tregs). ${ }^{11}$ Therefore, it has been suggested that the main criteria for the direction of the immune response - induction of immunity or tolerance - are the choice of DC population, maturation status, antigen dosage, and cytokine milieu. ${ }^{12}$

DCs comprise a heterogeneous cell population which can be divided into two main lineages, myeloid DC (mDC), also called conventional DC, and plasmacytoid (pDC). The latter produces large amounts of type I interferons (INF- $\alpha$, $-\beta$, and $-\omega)$ in response to viral infections, and are also known as natural interferon-producing cells (IPC). ${ }^{13,14}$ The $\mathrm{mDC}$ lineage consists of several cell subsets with different functions that can be found almost everywhere in the body. It is still a matter of debate to what extent cell populations characterized in mice correspond to populations identified in humans, ${ }^{15}$ but it is clear that DC subsets have distinct functions. In human peripheral blood, mainly three DC populations can be distinguished: $\mathrm{pDCs}$, characterized as

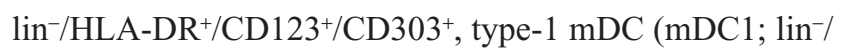
HLA-DR $\left.{ }^{+} / \mathrm{CD} 1 \mathrm{c}^{+}\right)$, and type- $2 \mathrm{mDC}\left(\mathrm{mDC} 2\right.$; lin $^{-} / \mathrm{HLA}^{-} \mathrm{DR}^{+} /$ $\left.\mathrm{CD} 141^{+}\right){ }^{16}$

In the intestines, DCs are present in the lamina propria of the small and large bowel, Peyer's patches (PPs) and mesenteric lymph nodes. DCs have an essential role in directing the balance between tolerance and immunity by cytokines and expression of costimulatory molecules. In the gut, particulate (insoluble) antigens are taken up in two ways: i) DCs can directly sample antigens by extending processes through the epithelium and into the lumen; and ii) $\mathrm{M}$ cells which overlie PPs can take up antigens and deliver them to subepithelial DCs. However, soluble antigens cross the epithelium through transcellular or paracellular routes, via brush border membrane and tight junctions, respectively. ${ }^{6,17}$ After sampling of antigen by DCs, they present them as peptide - major histocompatibility complex (MHC) complexes to effector T-cells in lymphoid organs.

The role of DCs in intestinal food hypersensitivity has not been evaluated fully. In the present pilot study, we wanted to investigate whether DC populations from patients with selfreported food hypersensitivity differ from those of healthy age- and gender-matched individuals, and their relationship with atopic disease. For this purpose, we enumerated the DC populations present in peripheral blood and analyzed monocyte-derived DCs with regard to their phenotype and $\mathrm{T}$ cell stimulatory capacity.

\section{Material and methods \\ Patients}

Ten consecutive patients referred to the Haukeland University Hospital because of abdominal complaints self-attributed to food hypersensitivity were included in the study. Organic diseases such as inflammatory bowel diseases (IBD), severe anaphylactic reactions, and pregnancy or lactation were exclusion criteria.

The patients went through an extensive clinical examination including both allergological and gastroenterological examinations as reported previously. ${ }^{3}$ Briefly, the allergological examination consisted of a full medical history including atopy, family history, suspected food items, serum levels of total and food-specific IgE, and skin prick tests using a panel of inhalant and food antigens (ALK; Abello, Hørsholm, Denmark). The serum levels of total and food-specific IgE were analyzed by ImmunoCap-System (Phadia, Uppsala, Sweden). Open provocation test and double-blind placebo-controlled food challenge test (DBPCFC) were performed by a dietician when indicated according to medical history and results of skin prick test and food-specific IgE levels in serum. The gastroenterological examination included a screening questionnaire based on the Rome II criteria for the diagnosis of IBS, ${ }^{18}$ and gastroscopy with gastric and duodenal biopsies to diagnose Helicobacter pylori infection and celiac disease, respectively. Stool tests were performed to exclude gastrointestinal infections.

The control group consisted of 10 gender- and agematched healthy controls without indication of allergic disease, including a history of atopy, recruited from the same geographical area as the patients. Blood $(40-50 \mathrm{~mL})$ 
was taken in heparin tubes from all patients and healthy controls. At the time of blood taking the patients were neither on an elimination diet nor being exposed to food challenge. All blood samples were processed within 2 hours.

The study was approved by the Western Norway Regional Committee for Medical Research Ethics and the subjects gave informed consent for the study.

\section{Enumeration of DC subsets in peripheral blood}

DC populations were stained with the Blood DC Enumeration kit (Miltenyi Biotec, Bergisch Gladbach, Germany), according to the manufacturer's instructions. Cells were analyzed on a BD FACSCanto I flow cytometer (BD Bioscience, San Jose, CA) and data analyses were performed using FlowJo software (Tree Star Inc., Ashland, OR).

\section{Ex vivo generation of monocyte-derived dendritic cells (moDCs)}

DCs were generated from monocytes isolated from heparinized blood from patients and gender- and age-matched healthy controls as described previously. ${ }^{19}$ Briefly, peripheral blood mononuclear cells were separated by density gradient centrifugation and the monocytes were then isolated by plastic adherence. The monocytes were cultured with interleukin- 4 (IL-4, 20 ng/mL; Immunotools, Friesoythe, Germany) and granulocyte macrophage colony-stimulating factor (100 ng/mL; Immunotools) in RP10 medium (RPMI 1640; Cambrex Bioscience, Verviers, Belgium) with 10\% FCS (PAA, Pasching, Austria); 100 units/mL penicillin and $100 \mu \mathrm{g} / \mathrm{mL}$ streptomycin (Sigma-Aldrich, St. Louis, MO) for 5-6 days to generate immature DC. Cytokines were replenished every 2-3 days. In some experiments, maturation of the cells was induced by addition of LPS on day 5 (100 ng/mL; Sigma-Aldrich, Oslo, Norway) for 24 hours.

\section{Immunostaining}

Phenotypic characterization of moDC was performed using monoclonal mouse anti-human CD1a-PE (NA1/34-HLK), CD14-FITC (UCHM1), HLA-DR-APC (HL-39), CD38Alexa Fluor 647 (AT13/5), CD40-FITC (LOB7/6), CD83-PE (HB15e), CD86-FITC (BU63) (AbD Serotec, Düsseldorf, Germany), CD80-APC (MEM-233; Immunotools), and CCR7-PE (R\&D systems, Abingdon, UK). The cells were incubated with titrated antibodies for 10 minutes at room temperature before they were washed and analyzed immediately on a FACSCanto I flow cytometer. All subsequent analyses were done using FlowJo software. One percent falsepositive events were accepted throughout the analyses.

\section{Mixed lymphocyte reaction}

Responding cells $\left(2 \times 10^{5}\right)$ from allogeneic nonadherent cells labeled with CFSE (Vybrant ${ }^{\circledR}$ CFDA SE Cell Tracer Kit; Invitrogen, Carlsbad, CA) were co-cultured with various numbers of DC $\left(2 \times 10^{4}\right.$ and $\left.5 \times 10^{4}\right)$ in 96-well flat-bottom microtiterplates (Greiner Bio-One $\mathrm{GmbH}$, Frickenhausen, Germany). CFSE intensity was measured on day 5 using a FACSCanto I flow cytometer. All subsequent analyses were done using FlowJo software.

\section{Cytokine determination}

Concentrations of IL-6, IL-10, and IL-12p70 in supernatants from moDC cultures were measured with commercially available two-site sandwich ELISAs from BioLegend (Uithoorn, The Netherlands) or R\&D Systems (Minneapolis, MN) according to the manufacturer's instructions.

\section{Statistical analysis}

Statistical analyses were performed by using GraphPad Prism 5.0 (GraphPad Prism Software Inc., La Jolla, CA). Data are presented as percentage of analyzed leukocytes. Values are expressed as the median, in some cases with interquartile ranges (IQR). A two-tailed Mann-Whitney $U$ test was used for comparison of groups. Correlation between total $\mathrm{IgE}$ and CD38 expression in patients was analyzed using Spearman's rank correlation test.

\section{Results Subjects characteristics}

Patients (six female, four male, age range 19-54 years, mean age 31 years) and controls (five female, five male, age range 20-57 years, mean age 34 years) were comparable with respect to age and gender.

In the patients, cow's milk (60\% of the patients) and wheat flour $(60 \%)$ were the most commonly suspected food item, and diarrhea (80\%) and abdominal pain (80\%) were the most common gastrointestinal symptoms, followed by bloating (50\%) and nausea (20\%).

The patients were divided into atopic and nonatopic subgroups, according to history, specific IgE in serum and skin prick tests. Atopic individuals were defined as having at least one positive skin prick test and/or elevated levels of specific $\mathrm{IgE}$ in serum to at least one allergen, as well as personal history of at least one of the following atopic symptoms: atopic dermatitis, asthma, or rhinoconjunctivitis. ${ }^{20}$ Five of 10 
patients were classified as having atopic disease. Four of those were sensitized to at least one inhalant allergen, as verified by skin prick test and/or serum-specific IgE whereas three of the atopic patients were sensitized to at least one food allergen. Birch (80\%) and timothy grass (80\%) were the most common inhalant allergen, while wheat flour $(60 \%)$ and peanuts $(40 \%)$ were the most common food allergen. Of the atopic patients, four had atopic dermatitis $(80 \%)$, three had rhinoconjunctivitis (60\%), and one had asthma (20\%). Total serum IgE levels were significantly higher in the atopic patients (median, $141 \mathrm{kU} / \mathrm{L}$; IQR, 45-554 kU/L) than in the nonatopic patients (median, $19 \mathrm{kU} / \mathrm{L}$; IQR, 7-32 kU/L; $P<0.008$ ).

DBPCFC was positive in one patient $(10 \%$ of the whole study population), which indicates non-IgE-mediated allergy or nonallergic food hypersensitivity. None of the patients had IgE-mediated food allergy as confirmed by both skin prick test, food-specific IgE levels in serum, and DBPCFC with the same food item. Nine of 10 patients (90\%) had gastrointestinal symptoms consistent with IBS, according to the Rome II criteria.

\section{Dendritic cell enumeration and phenotyping}

Using the Blood DC Enumeration kit (Miltenyi Biotec), we could not detect differences in the peripheral blood DC populations between patients with food hypersensitivity and healthy controls (Figure 1). The subgroups, pDCs, mDC1, and $\mathrm{mDC} 2$, were similar in patients and in controls.

Concerning the phenotype of moDCs, we analyzed DC markers (CD14- and CD1 $\left.{ }^{+}\right)$, costimulatory molecules (CD40, CD80, and CD86), DC maturation markers (CD83 and $\mathrm{MHC}$ class II), and molecules shown to be important for migration of DC (CCR7 and CD38) in unstimulated and lipopolysaccharide (LPS)-stimulated cells. We could not detect any obvious differences of expression levels between patients and controls, even if the expression of MHC class II and CD83 was slightly increased in both unstimulated and LPS-stimulated moDCs of patients (Figure 2).

\section{Dendritic cells in atopic disease}

Comparing DC populations in the peripheral blood (pDCs, $\mathrm{mDC} 1$, and $\mathrm{mDC} 2$ ) for atopic and nonatopic subgroups yielded no obvious differences between the subgroups (data not shown).

Concerning the phenotype, LPS-stimulated moDCs from atopic patients expressed significantly more CD38 compared to nonatopic patients analyzed by median fluorescence intensity (atopic $=1055$, nonatopic $=405 ; P=0.03$; Figure 3B) even though the percentage positive cells was not significantly different $($ atopic $=99 \%$, nonatopic $=79 \%$; Figure $3 \mathrm{~A}$ ). MHC class II and CD83 expression did not differ significantly between atopic and nonatopic patients, either analyzed as percentage

A

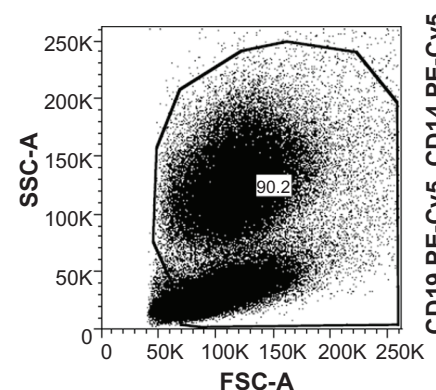

FSC-A

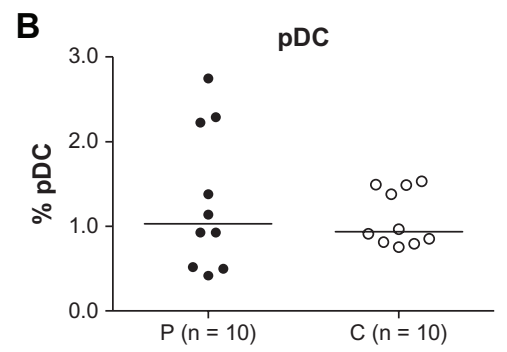

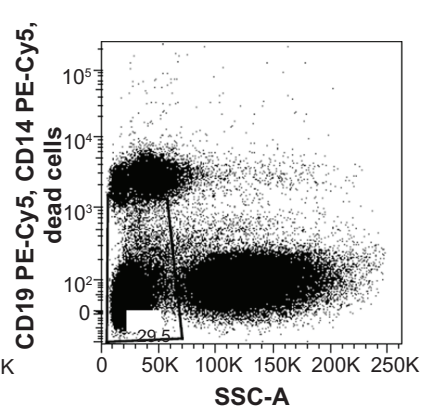
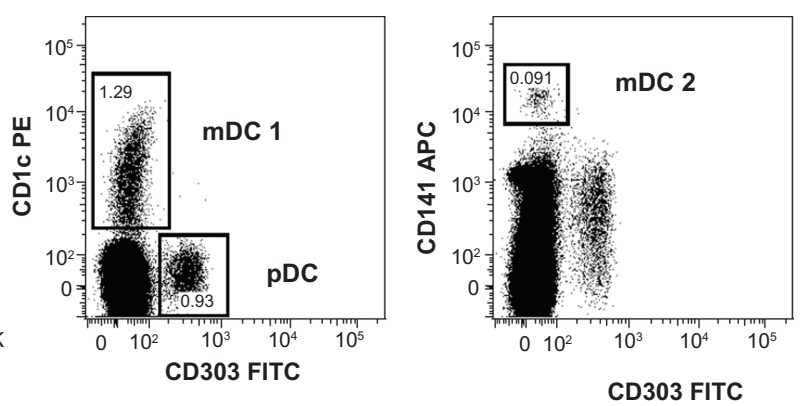

mDC1

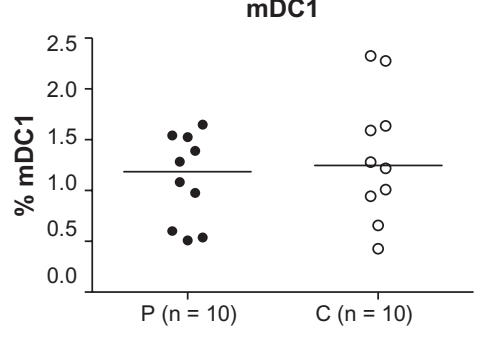

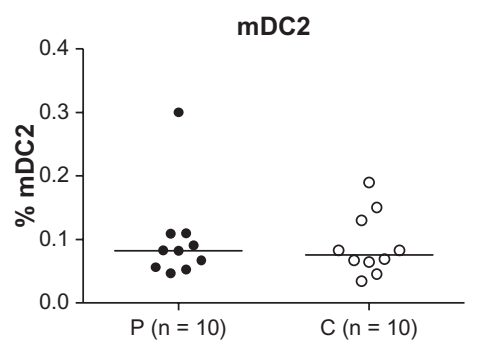

Figure I Patients with self-reported food hypersensitivity have similar percentage of peripheral blood DC populations compared to healthy controls. DC subsets among leukocytes in peripheral blood were analyzed using the Blood DC Enumeration kit (Miltenyi Biotec) on freshly heparinized whole blood samples. A) Representative plots showing the gating strategy for $\mathrm{pDC}, \mathrm{mDCl}$, and $\mathrm{mDC2}$. B) Scatter plots with median lines.

Abbreviations: $\mathrm{C}$, controls, open circle, $\mathrm{n}=10$; DC, dendritic cells; mDCI, type-I myeloid DC; mDC2, type-2 myeloid DC; $\mathrm{P}$, patients, filled circle, $\mathrm{n}=10$; pDC, plasmacytoid DC. 
A

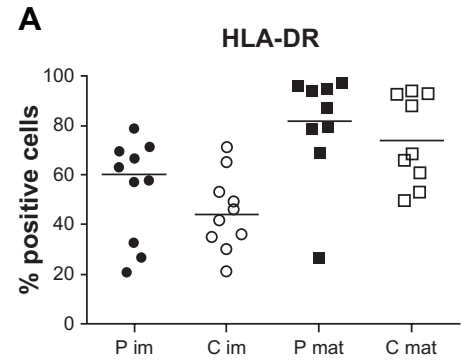

B

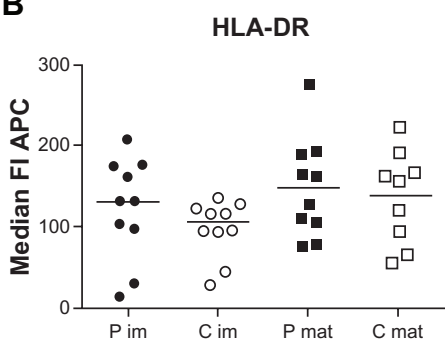

CD83

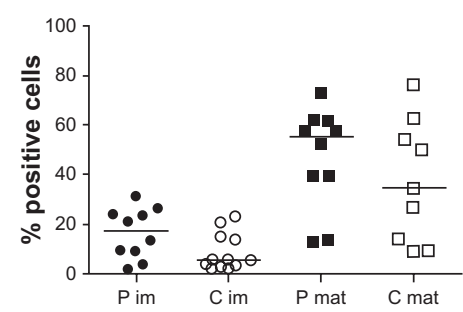

CD83

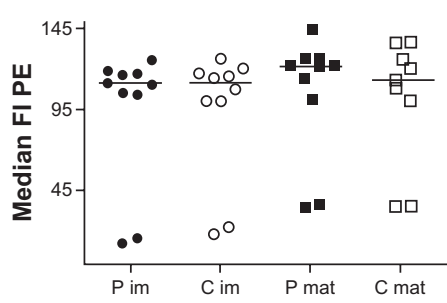

CD38

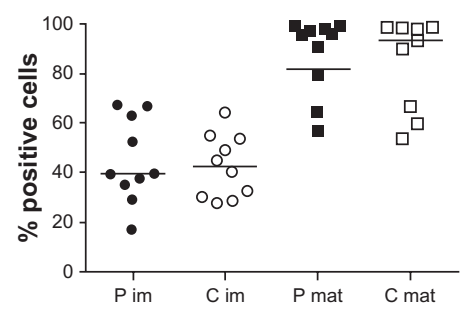

CD38

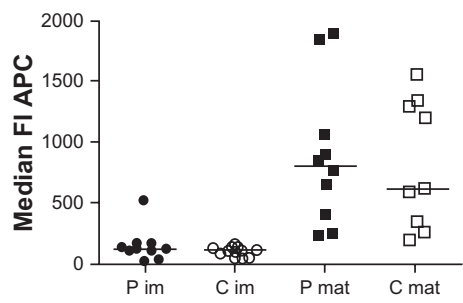

Figure 2 Monocyte-derived dendritic cells (moDCs) from patients with self-reported food hypersensitivity have similar phenotypes as moDCs from healthy controls. Expression of MHC class II, CD83 and CD38 of immature (im, $n=10$ each) and LPS-stimulated (mat, $n=9$ each) moDCs from patients (P, filled symbols) and controls (C, open symbols) did not differ significantly, either analyzed as percentage of positive cells $(\mathbf{A})$ or as median fluorescence intensity (B). Analysis performed by flow cytometry accepting I\% false positive events throughout.

Abbreviations: LPS, lipopolysaccharide; MHC, major histocompatibility complex.

of positive cells or median fluorescence intensity (Figure 3). The same results regarding median fluorescence intensity were obtained when gating on marker positive cells only. Interestingly, CD38 expression was significantly correlated to total serum IgE levels in patients $(r=0.89, P=0.001)$.

\section{Functional analyses}

We next analyzed the amount of IL-6, IL-10, and IL-12p70 in the supernatants of the moDC cultures. As expected, no IL-6 or IL-12p70 were produced from immature moDCs. The median level of IL- 6 and IL-12p70 produced from LPS-stimulated moDCs from patients and controls was similar (IL-6: patients $741 \mathrm{pg} / \mathrm{mL}$, controls $708 \mathrm{pg} / \mathrm{mL}$; IL-12p70: patients $91.26 \mathrm{pg} / \mathrm{mL}$, controls $109.3 \mathrm{pg} / \mathrm{mL}$; Figures 4A and B). LPS stimulation increased IL-10 production in both controls and patients (immature patients $47.44 \mathrm{pg} / \mathrm{mL}$, immature controls $34.83 \mathrm{pg} / \mathrm{mL}$, LPS-stimulation patients $189.9 \mathrm{pg} / \mathrm{mL}$, LPS-stimulation controls $146.6 \mathrm{pg} / \mathrm{mL}$, Figure 4C). When we divided the patients into atopic and nonatopic subgroups, we found that LPS-stimulated moDCs from atopic patients produced significantly more IL-10 compared to cells from nonatopic patients (atopic $410.1 \mathrm{pg} / \mathrm{mL}$, nonatopic $129.9 \mathrm{pg} / \mathrm{mL}$; $P=0.02$; Figure $4 \mathrm{D}$ )

Analyzing the $\mathrm{T}$ cell stimulatory capacity of the generated DC populations in an allogeneic MLR, we detected no obvious differences between cells from patients and controls, either analyzed together or divided into atopic and nonatopic (data not shown).

\section{Discussion}

A relationship between food intolerance and IBS is well recognized. Up to $70 \%$ of the IBS patients report adverse reactions to one or more food items, and almost $90 \%$ of IBS patients show evidence of immune activation. ${ }^{21,22}$ Previous immunological studies indicate important functions of $\mathrm{T}$ and B cells in patients with IBS, but we are unable to find information about DCs. ${ }^{23}$ In the present study, most of the patients $(90 \%)$ had IBS-like gastrointestinal symptoms in response to food intake. Recently, we have shown that more than $60 \%$ of patients with self-reported food hypersensitivity have indications of atopic disease and abnormally high numbers of "IgE-armed" mast cells in their duodenal mucosa which was correlated to serum total IgE levels. ${ }^{24}$ Patients with self-reported food hypersensitivity also had increased concentration of B cell-activation factor (BAFF) in gut lavage fluid and blood, ${ }^{25}$ indicating intestinal immune activation in many of these patients. The driving force is not known. BAFF is mainly produced and secreted by myeloid cells such as DCs, macrophages, and monocytes. If intestinal allergens are involved, intestinal DCs may play a role. We therefore analyzed various DC populations in the present study.

A vast number of antigens are encountered by the intestine, which needs to distinguish between invading 
A

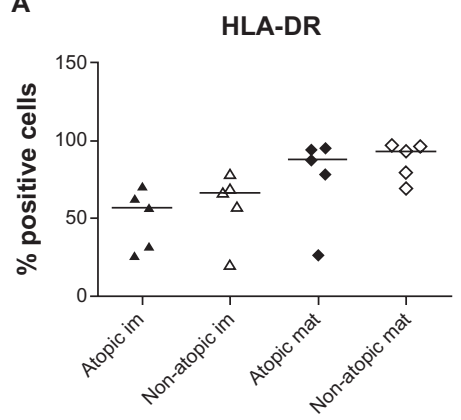

B

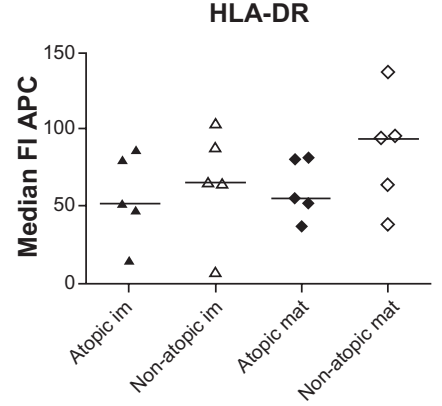

CD83

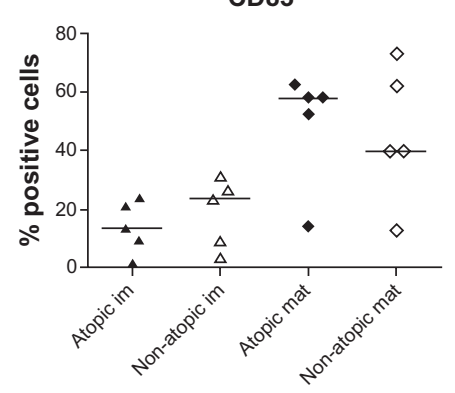

CD83

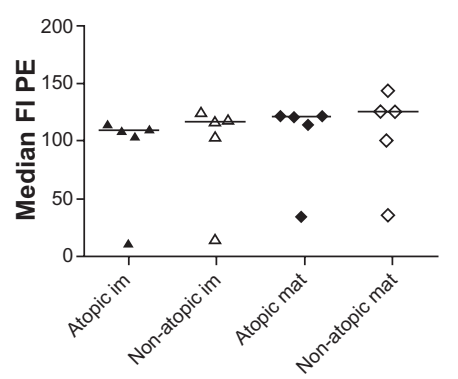

CD38
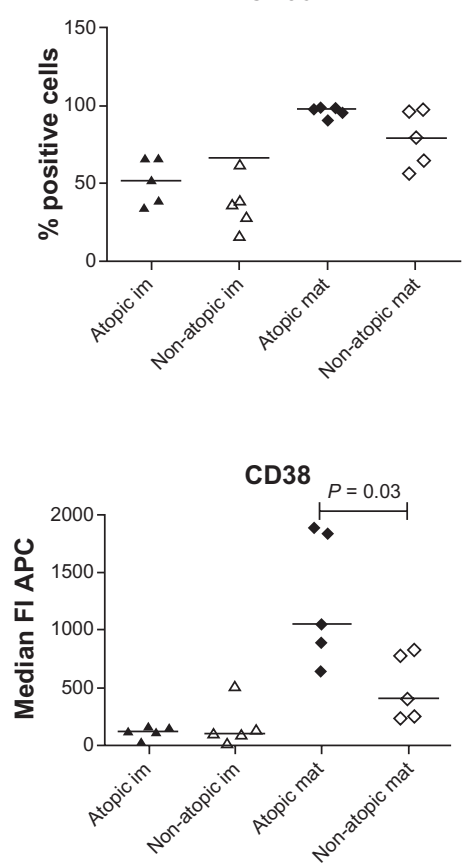

Figure 3 LPS-stimulated moDCs from atopic patients express significantly more CD38 compared to similarly stimulated moDCs from nonatopic patients. Surface expression of MHC class II, CD83 and CD38 was analyzed by flow cytometry on immature (im, $n=5$ each) and LPS-stimulated (mat, $n=4$ each) moDCs. MHC class II and CD83 expression did not differ significantly between atopic (filled symbols) and nonatopic (open symbols) patients, either analyzed as percentage of positive cells (A) or as median fluorescence intensity (B). Analysis performed as described in Figure 2.

Abbreviations: LPS, lipopolysaccharide; moDCs, monocyte-derived dendritic cells; MHC, major histocompatibility complex.

pathogens and other nonself antigens introduced as part of an individual's diet. Some DC populations localized in the lamina propria of the small intestine and in mesenteric lymph nodes have been shown to be important in inducing Treg and nonreactivity in the steady state. In patients with active IBD, alterations of DC populations in both peripheral blood and intestinal mucosa have been described and an involvement of DCs in the imbalance between Treg and Th17 cells has been proposed. ${ }^{26,27}$ Furthermore, peripheral blood DCs from IBD patients expressed more costimulatory molecules ${ }^{28}$ and displayed aberrant response upon LPS stimulation..$^{29}$ In the present pilot study, patients with self-reported food hypersensitivity had normal DC populations in peripheral blood and the phenotype of moDCs was similar in patients and healthy controls. However, atopic and nonatopic patients showed significantly different CD38 expression on LPS-stimulated moDCs. The multifunctional surface marker CD38 is a receptor with ectoenzymatic activity. It catalyzes the production of adenosine diphosphate (ADP)-ribose (ADPR) and cyclic ADPR from $\mathrm{NAD}^{+}$resulting in calcium influx. In animal models, CD38-deficient mice are defective in mounting innate and adaptive immune responses and have impaired migration of neutrophils, monocytes, and DCs. ${ }^{30,31}$ In addition, these mice exhibit strongly reduced allergen-induced airway hyperresponsiveness, which is accompanied by a decrease in hallmarks of pulmonary inflammation, as well as Th2 cytokine levels (IL-4, IL-5, and IL-13). Concerning CD38 expression on moDCs, Fedele and colleagues showed that CD38 is highly expressed on monocytes, downregulated during DC development, and upregulated upon maturation of moDCs. ${ }^{32}$ Moreover, CD38 has been shown to be important for migration, survival, and Th1 immune responses of mature moDCs. ${ }^{33}$ However, not much is known about the consequences of CD38 overexpression on DCs.

DCs play an important role in allergic airway disease. Increased numbers of airway mucosal DCs are found in both allergic rhinitis and asthma, and these DCs express the high affinity IgE receptor. Treatment of asthma patients with inhaled steroids predictably leads to a decrease in airway DC numbers. Another work showed a rapid disappearance of myeloid DC precursors from the circulation, but recruitment of these cells into the airway mucosa after short-term allergen inhalation. ${ }^{34}$ Recruitment of DCs within the intestinal mucosa during inflammation was also shown in patients with IBD. ${ }^{35,36}$ Therefore, mucosal DC characteristics in response to food challenge should be investigated in future studies in patients with self-reported food hypersensitivity. 
A

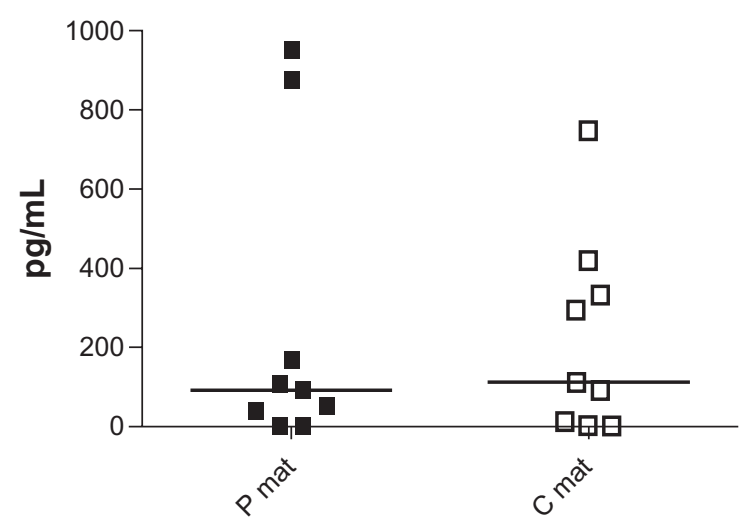

C

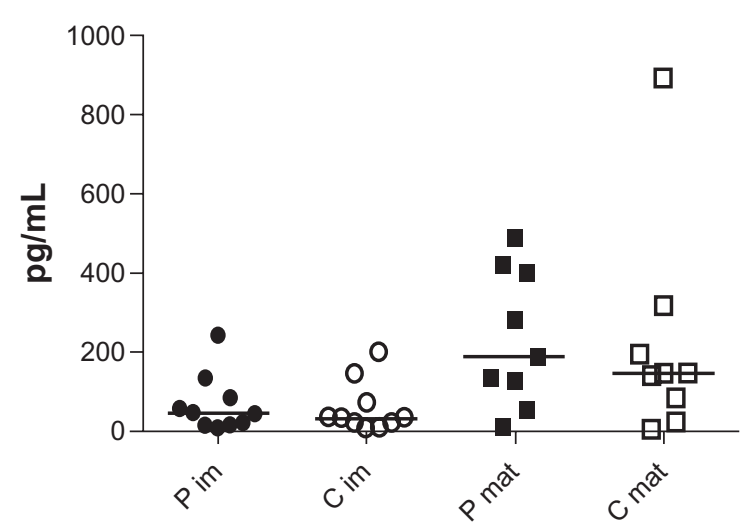

B

IL-6

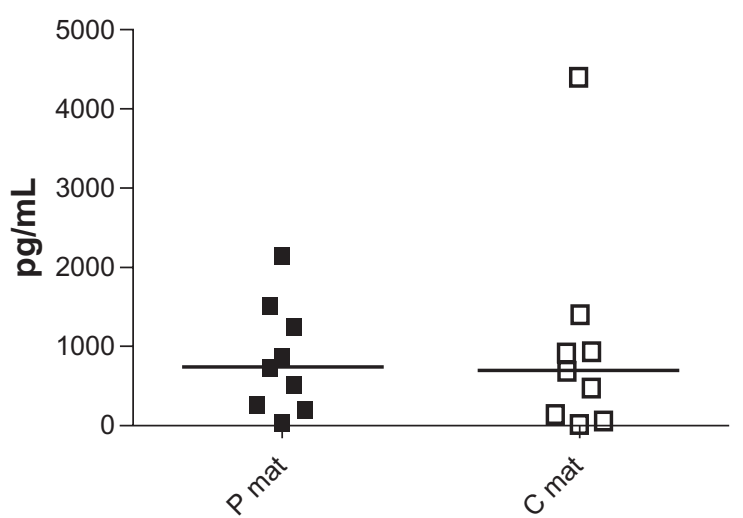

C

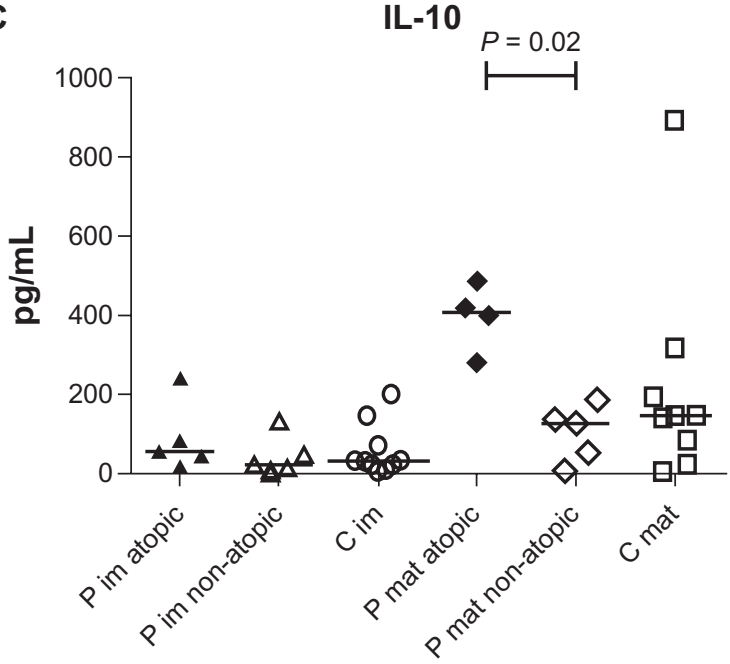

Figure 4 Monocyte-derived dendritic cells (moDCs) from atopic patients produce significantly more IL-I0 after LPS stimulation than stimulated moDCs from nonatopic patients or healthy controls. Cytokine production was analyzed by ELISA. A) IL- I 2p70 production from LPS-stimulated moDCs from patients (P mat, filled square; $n=9)$ and controls ( $C$ mat, open square; $n=9$ ) did not differ significantly. B) No difference in IL-6 production from LPs-stimulated moDcs from patients (P mat, filled square; $n=9)$ and controls ( $\mathrm{C}$ mat, open square; $\mathrm{n}=9$ ) was observed. $C)$ Immature moDCs from patients ( $\mathrm{P}$ im, filled circle; $\mathrm{n}=10)$ and controls $(\mathrm{C}$ im, open circle; $\mathrm{n}=\mathrm{I0})$ produce similar amounts of IL-10. LPS stimulation (mat) increased IL-10 production in both patients (filled square; $n=9$ ) and controls (open square; $n=9$ ). $\mathbf{D}$ ) Immature moDCs from atopic (filled triangle; $n=5$ ) and nonatopic patients (open triangle; $n=5$ ) secreted similar amounts of IL- 10 as cells from controls (open circle; $n=10$ ). LPS-stimulated moDCs from atopic patients (filled diamond; $n=4$ ) secreted significantly more IL- 10 than LPS-stimulated moDCs from nonatopic patients (open diamond; $n=5$ ).

Abbreviations: IL, interleukin; LPS, lipopolysaccharide; moDCs, monocyte-derived dendritic cells.

In conclusion, even though only a limited number of patients were included in the present pilot study, atopic patients had significantly higher CD38 expression on LPS-stimulated moDCs compared to nonatopic patients. Moreover, LPS stimulated moDCs from atopic patients produced significantly more IL-10 compared to nonatopic patients. This is an interesting finding as it has been shown previously that in inflammatory conditions, IL-10 is responsible for the expression of Foxp3 in Tregs. ${ }^{37}$ It is still a matter of debate, however, whether an increase in IL-10 leads to an increase in IgE production. ${ }^{38}$ Interestingly, CD38 expression was correlated to total serum IgE levels in patients. These findings support the notion of immune activation in some patients with self-reported food hypersensitivity. Further studies including DC populations in the intestinal mucosa and in response to food challenge are warranted.

\section{Acknowledgments/Disclosure}

We thank all patients and healthy controls for kindly donating blood for our study and Dagny Ann Sandnes and Marie Karlsen for excellent technical help. The authors have been financially supported by the Faculty of Medicine and Dentistry at the University of Bergen, the Strategic Research Program at Helse Bergen, the Western Norway Health Authority, the Broegelmann Foundation, the Norwegian Cancer Society and Bergen Research Foundation. The authors report no conflicts of interest in this work. 


\section{References}

1. Young E, Stoneham MD, Petruckevitch A, Barton J, Rona R. A population study of food intolerance. Lancet. 1994;343:1127-1130.

2. O'Leary PF, Shanahan F. Food allergies. Curr Gastroenterol Rep. 2002;4:373-382.

3. Arslan G, Kahrs GE, Lind R, Froyland L, Florvaag E, Berstad A. Patients with subjective food hypersensitivity: the value of analyzing intestinal permeability and inflammation markers in gut lavage fluid. Digestion. 2004;70:26-35.

4. Gibson PR, Shepherd J. Evidence-based dietary management of functional gastrointestinal symptoms: The FODMAP approach. J Gastroenterol Hepatol. 2010;25:252-258.

5. Asero R, Ballmer-Weber BK, Beyer K, et al. IgE-mediated food allergy diagnosis: Current status and new perspectives. Mol Nutr Food Res. 2007;51:135-147.

6. Burks AW, Laubach S, Jones SM. Oral tolerance, food allergy and immunotherapy: Implications for future treatment. J Allergy Clin Immunol. 2008;121:1344-1350.

7. Banchereau J, Steinman RM. Dendritic cells and the control of immunity. Nature. 1998;392:245-252.

8. Heath WR, Carbone FR. Cross-presentation, dendritic cells, tolerance and immunity. Annu Rev Immunol. 2001;19:47-64.

9. Steinman RM, Hawiger D, Nussenzweig MC. Tolerogenic dendritic cells. Annu Rev Immunol. 2003;21:685-711.

10. Hawiger D, Inaba K, Dorsett Y, et al. Dendritic cells induce peripheral $\mathrm{T}$ cell unresponsiveness under steady state conditions in vivo. $J \operatorname{Exp}$ Med. 2001;194:769-779.

11. Yamazaki S, Iyoda T, Tarbell K, et al. Direct expansion of functional CD25+CD4+ regulatory T cells by antigen-processing dendritic cells. J Exp Med. 2003;198:235-247.

12. Rutella S, Danese S, Leone G. Tolerogenic dendritic cells: cytokine modulation comes of age. Blood. 2006;108:1435-1440.

13. Siegal FP, Kadowaki N, Shodell M, et al. The nature of the principal type 1 interferon-producing cells in human blood. Science. 1999;284: 1835-1837.

14. Liu YJ. IPC: professional type 1 interferon-producing cells and plasmacytoid dendritic cell precursors. Annu Rev Immunol. 2005;23:275-306.

15. Naik SH. Demystifying the development of dendritic cell subtypes, a little. Immunol Cell Biol. 2008;86:439-452.

16. Banchereau J, Klechevsky E, Schmitt N, Morita R, Palucka K, Ueno H. Harnessing human dendritic cell subsets to design novel vaccines. Ann NY Acad Sci. 2009;1174:24-32.

17. Chehade M, Mayer L. Oral tolerance and its relation to food hypersensitivities. J Allergy Clin Immunol. 2005;115:3-12.

18. Thompson WG, Longstreth GF, Drossman DA, et al. Functional bowel disorders and functional abdominal pain. Gut. 1999;45(Suppl 2); II43-II47.

19. Appel S, Rupf A, Weck MM, et al. Effects of imatinib on monocytederived dendritic cells are mediated by inhibition of nuclear factor-kappaB and Akt signaling pathways. Clin Cancer Res. 2005;11:1928-1940.

20. Johansson SGO, Bieber T, Dahl R, et al. Revised nomenclature for allergy for global use: Report of the nomenclature review committee of the world allergy organization, October 2003. J Allergy Clin Immunol. 2004;113:832-836.

21. Monsbakken KW, Vandvik PO, Farup PG. Perceived food intolerance in subjects with irritable bowel syndrome: etiology, prevelance and consequences. Eur J Clin Nutr. 2006;60:667-672.

International Journal of General Medicine

\section{Publish your work in this journal}

The International Journal of General Medicine is an international, peer-reviewed open-access journal that focuses on general and internal medicine, pathogenesis, epidemiology, diagnosis, monitoring and treatment protocols. The journal is characterized by the rapid reporting of reviews, original research and clinical studies across all disease areas.
22. Chadwick VS, Chen WX, Shu D, et al. Activation of the mucosal immune system in irritable bowel syndrome. Gastroenterology. 2002; 122:1778-1783.

23. Ohman L, Lindmark AC, Isaksson S, et al. B-cell activation in patients with irritable bowel syndrome (IBS). Neurogastroenterol Motil. 2009; 21:644-650.

24. Lillestøl K, Helgeland L, Lied GA, et al. Indication of "atopic bowel" in patients with self-reported food hypersensitivity. Aliment Pharmacol Ther. 2010;31:1112-1122.

25. Lied GA, Lillestøl K, Valeur J, Berstad A. Intestinal B cell-activating factor (BAFF): An indication of non-IgE-mediated hypersensitivity reactions to food? Aliment Pharmacol Ther. 2010;32:66-73.

26. Rescigno M, Di SA. Dendritic cells in intestinal homeostasis and disease. J Clin Invest. 2009;119:2441-2450.

27. Baumgart DC, Metzke D, Schmitz J, et al. Patients with active inflammatory bowel disease lack immature peripheral blood plasmacytoid and myeloid dendritic cells. Gut. 2005;54:228-236.

28. Vuckovic S, Florin TH, Khalil D, et al. CD40 and CD86 upregulation with divergent CMRF44 expression on blood dendritic cells in inflammatory bowel diseases. Am J Gastroenterol. 2001;96: 2946-2956.

29. Baumgart DC, Thomas S, Przesdzing I, et al. Exaggerated inflammatory response of primary human myeloid dendritic cells to lipopolysaccharide in patients with inflammatory bowel disease. Clin Exp Immunol. 2009;157(3):423-436.

30. Partida-Sanchez S, Goodrich S, Kusser K, Oppenheimer N, Randall TD, Lund FE. Regulation of dendritic cell trafficking by the ADP-ribosyl cyclase CD38: impact on the development of humoral immunity. Immunity. 2004;20:279-291.

31. Partida-Sanchez S, Cockayne DA, Monard S, et al. Cyclic ADP-ribose production by CD38 regulates intracellular calcium release, extracellular calcium influx and chemotaxis in neutrophils and is required for bacterial clearance in vivo. Nat Med. 2001;7:1209-1216.

32. Fedele G, Frasca L, Palazzo R, Ferrero E, Malavasi F, Ausiello CM. CD38 is expressed on human mature monocyte-derived dendritic cells and is functionally involved in CD83 expression and IL-12 induction. Eur J Immunol. 2004;34:1342-1350.

33. Frasca L, Fedele G, Deaglio S, et al. CD38 orchestrates migration, survival, and Th1 immune response of human mature dendritic cells. Blood. 2006;107:2392-2399.

34. Upham JW. The role of dendritic cells in immune regulation and allergic airway inflammation. Respirology. 2003;8:140-148.

35. Middel P, Raddatz D, Gunawan B, Haller F, Radzun HJ. Increased number of mature dendritic cells in Crohn's disease: evidence for a chemokine mediated retention mechanism. Gut. 2006;55:220-227.

36. Silva MA. Intestinal dendritic cells and epithelial barrier dysfunction in Crohn's disease. Inflamm Bowel Dis. 2009;15:436-453.

37. Murai M, Turovskaya O, Kim G, et al. Interleukin 10 acts on regulatory $\mathrm{T}$ cells to maintain expression of the transcription factor Foxp3 and suppressive function in mice with colitis. Nat Immunol. 2009;10: 1178-1184.

38. Kobayashi N, Nagumo H, Agematsu K. IL-10 enhances B-cell IgE synthesis by promoting differentiation into plasma cells, a process that is inhibited by CD27/CD70 interaction. Clin Exp Immunol. 2002;129:446-452.

A key focus is the elucidation of disease processes and management protocols resulting in improved outcomes for the patient. The manuscript management system is completely online and includes a very quick and fair peer-review system. Visit http://www.dovepress.com/ testimonials.php to read real quotes from published authors. 